\title{
Photoinduced ordering and anchoring properties of azo-dye films
}

\author{
Alexei D. Kiselev, ${ }^{*}$ Vladimir Chigrinov, ${ }^{\dagger}$ and Dan Ding Huang \\ Hong Kong University of Science and Technology, Clear Water Bay, Kowloon, Hong Kong
}

(Received 23 July 2005; published 14 December 2005)

\begin{abstract}
We study both theoretically and experimentally the anchoring properties of photoaligning azo-dye films in contact with a nematic liquid crystal depending on the photoinduced ordering of azo-dye molecules. In the mean field approximation, we found that the bare surface anchoring energy depends linearly on the azo-dye order parameter and the azimuthal anchoring strength decays to zero in the limit of vanishing photoinduced ordering. From the absorption dichroism spectra measured in azo-dye films that are prepared from an azo-dye derivative with polymerizable terminal groups we obtain the dependence of the dichroic ratio on the irradiation dose. We also measure the polar and azimuthal anchoring strengths in nematic liquid crystal (NLC) cells aligned by the azo-dye films and derive the anchoring strengths as functions of the dichroic ratio, which is proportional to the photoinduced order parameter. Although linear fitting of the experimental data for both anchoring strengths gives reasonable results, it, predicts vanishing of the azimuthal anchoring strength at some nonzero value of the azo-dye order parameter, in contradiction with theory. By using a simple phenomenological model we show that this discrepancy can be attributed to the difference between the surface and bulk order parameters in the films. The measured polar anchoring energy is found to be an order of magnitude higher than the azimuthal strength. Our theory suggests that the quadrupole term of the spherical harmonics expansion for the azo-dye-NLC intermolecular potential might be of importance for the understanding of this difference.
\end{abstract}

DOI: 10.1103/PhysRevE.72.061703

PACS number(s): 61.30.Hn, 42.70.Gi, 78.40.-q

\section{INTRODUCTION}

When a nematic liquid crystal (NLC) is brought into contact with an anisotropic substrate, the energy of the NLC molecules in the interfacial layer and thus the surface tension (the excess free energy per unit area) will be orientation dependent. The anisotropic part of the surface tension-the socalled anchoring energy-gives rise to the phenomenon known as anchoring, i.e., surface induced alignment of the nematic director along the vector of preferential orientation referred to as the easy axis.

Over the past few decades anchoring properties of NLCs have been the subject of intense studies for both technological and more fundamental reasons. There are a number of surface ordering and anchoring transitions that were observed experimentally and studied using different theoretical approaches (see, e.g., Refs. [1-3] for reviews).

Technologically, producing substrates with anisotropic anchoring properties is of vital importance in the fabrication of liquid crystal electro-optic devices. The traditional technique widely used to align liquid crystal display cells involves mechanical rubbing of aligning layers. This method, however, has the well known difficulties related to physical damage, impurities, dust contamination, and generation of electrostatic charge [4].

An alternative photoalignment technique avoiding the drawbacks of the mechanical surface treatment was suggested in Refs. [5-7]. It uses linearly polarized ultraviolet

\footnotetext{
*Present address: Institute of Physics of NASU, prospekt Nauki 46, 03028 Kyïv, Ukraine. Email address: kisel@mail.cn.ua

†Email address: eechigr@ust.hk
}

(uv) light to induce anisotropy of the angular distribution of molecules in a photosensitive film [8].

The photoalignment has been extensively studied in a number of different polymer systems such as dye doped polymer layers [5,9], cinnamate polymer derivatives [6,7,10-12], and side chain azopolymers [13-19]. Light induced ordering in the photosensitive materials, though not understood very well, can occur by a variety of photochemically induced processes. These typically may involve such transformations as photoisomerization, cross linking, photodimerization, and photodecomposition (a recent review can be found in Ref. [20]).

Films containing photochemically stable azo-dye structures (azobenzene sulfuric dyes) were recently studied as new photoaligning materials for NLC cells [21,22]. In Ref. [22], it was found that, owing to the high degree of the photoinduced ordering, these films used as aligning substrates are characterized by anchoring energy strengths comparable to those of rubbed polyimide films. For these materials, the voltage holding ratio and thermal stability of the alignment turned out to be high. In addition, durability against light exposure and moisture can be improved using the azo-dye monomer which can be polymerized by heating after photoalignment. The azo-dye films are thus promising materials for applications in liquid crystal devices.

The kinetics of the photoinduced anisotropy in the chemically stable azo-dye layers was studied in Ref. [23] using the diffusion model. By contrast with the models for azopolymers discussed in Ref. [24], this model assumes that photochemical mechanisms such as photoisomerization are suppressed and the process of light induced reorientation can be described as a diffusion motion of the dye molecules under the action of polarized light. 
In this paper the dependence of the surface anchoring strengths on the photoinduced anisotropy will be the subject of our primary interest. More specifically, we examine the effects of the photoinduced ordering in azo-dye films on the polar and azimuthal anchoring energies. The key point underlying our study is that the photoalignment technique provides a means for controlling the photoinduced ordering that affects anchoring properties of photoaligning layers by changing ordering of azo-dye molecules at the surface and thus the surface anchoring strengths.

Recently, the anchoring properties of aligning photopolymer layers in relation to the photoinduced ordering were studied experimentally in Ref. [25]. The relationship between the rubbing strength and the azimuthal anchoring energy was discussed in Ref. [26].

The photopolymer-NLC interface was also described theoretically in Refs. $[27,28]$ using a modified version of the variational mean field approach which is also known as the Maier-Saupe theory. By contrast, azo-dye films have not yet received a proper attention and we intend to fill the gap.

The paper is organized as follows. In Sec. II we apply the mean field theoretical approach [29-31] to express the surface anchoring energy in terms of the tensorial order parameters which characterize angular distribution of the azo-dye and NLC molecules at the interfacial boundary surface. The general result is then used to derive the expressions for the azimuthal and polar anchoring strengths that, in addition to the order parameters, depend on the harmonics of the intermolecular potentials.

Experimental details are given in Sec. III. The polymerizable azo-dye monomer SDA-2 was used to prepare the photoaligning layers. Absorption dichroism spectra were measured in the films irradiated with linearly polarized uv light at various irradiation doses. Anchoring energy measurements were performed in NLC cells where the NLC is sandwiched between glass plates coated with azo-dye film.

In Sec. IV we present the experimental results and apply the theory of Sec. II to interpret the data. Discussion and concluding remarks are given in Sec. V. Details on some technical results are relegated to Appendixes A and B.

\section{THEORY}

In this section we begin by introducing general notation and apply the mean field approach to express the Landau-de Gennes surface free energy in terms of both azo-dye and NLC order parameters. Expressions for the azimuthal and polar anchoring strengths, $W_{\phi}$ and $W_{\theta}$, are then derived from the orientationally dependent part of the surface energy in Sec. II C. In the concluding part of this section we consider the effects of spatial variations of the azo-dye order parameter using a simple model formulated in Sec. II D.

\section{A. Order parameter and dichroic ratio}

Assuming that the unit vector $\hat{\mathbf{u}}=(\sin \theta \cos \phi$, $\sin \theta \sin \phi, \cos \theta)$ directed along the long molecular axis defines the orientation of a molecule in both the azo-dye film and NLC cell, quadrupolar orientational ordering of the mol- ecules can be characterized using the traceless symmetric second-rank tensor [32]

$$
\mathbf{Q}(\hat{\mathbf{u}})=(3 \hat{\mathbf{u}} \otimes \hat{\mathbf{u}}-\mathbf{I}) / 2,
$$

where $\mathbf{I}$ is the identity matrix. The dyadic (1) averaged over orientations of molecules with the one-particle distribution function $\rho_{\alpha}(\mathbf{r}, \hat{\mathbf{u}})$, describing the orientation density profile of the azo-dye $(\alpha=\mathrm{A})$ and NLC $(\alpha=\mathrm{N})$ molecules, is proportional to the order parameter tensor $\mathbf{S}_{\alpha}(\mathbf{r})$

$$
\int \rho_{\alpha}(\mathbf{r}, \hat{\mathbf{u}}) \mathbf{Q}(\hat{\mathbf{u}}) d \hat{\mathbf{u}}=\rho_{\alpha}(\mathbf{r}) \mathbf{S}_{\alpha}(\mathbf{r}),
$$

where $\quad d \hat{\mathbf{n}} \equiv \sin \theta d \theta d \phi, \quad \rho_{\alpha}(\mathbf{r}, \hat{\mathbf{u}})=\rho_{\alpha}(\mathbf{r}) f_{\alpha}(\mathbf{r}, \hat{\mathbf{u}}), \quad \rho_{\alpha}(\mathbf{r})$ $=\int \rho_{\alpha}(\mathbf{r}, \hat{\mathbf{u}}) d \hat{\mathbf{u}}$ is the density profile, and $f_{\alpha}(\mathbf{r}, \hat{\mathbf{u}})$ is the normalized angular distribution. The general expression for the order parameter is given in Appendix A [see Eq. (A15)] along with technical details on the technique of irreducible tensors.

Now we dwell briefly on the relation between the order parameter $\mathbf{S}_{\mathrm{A}}$ characterizing the orientational distribution of azo-dye molecules $f_{\mathrm{A}}(\hat{\mathbf{u}})$ and the absorption dichroic ratio

$$
R=\frac{D_{\|}-D_{\perp}}{D_{\|}+2 D_{\perp}},
$$

where $D_{\|}\left(D_{\perp}\right)$ is the absorption coefficient measured for a testing beam linearly polarized parallel (perpendicular) to the polarization vector of the activating uv light which is directed along the $x$ axis, $\mathbf{E}_{\mathrm{ex}}=E_{\mathrm{ex}} \hat{\mathbf{x}}$. We shall also assume that the testing and the pumping waves are both propagating along the $z$ axis, which is normal to the film substrate.

When the absorption tensor of an azo-dye molecule is uniaxially anisotropic with $\sigma_{i j}(\hat{\mathbf{u}})=\sigma_{\perp} \delta_{i j}+\left(\sigma_{\|}-\sigma_{\perp}\right) u_{i} u_{j}$, its orientational average takes the following matrix form:

$$
\begin{gathered}
\langle\boldsymbol{\sigma}\rangle=\left(\sigma_{\mathrm{av}} \mathbf{I}+2 \Delta \sigma \mathbf{S}_{\mathrm{A}}\right) / 3, \\
\sigma_{\mathrm{av}}=\sigma_{\|}+2 \sigma_{\perp}, \quad \Delta \sigma=\sigma_{\|}-\sigma_{\perp},
\end{gathered}
$$

where the angular brackets $\langle\cdots\rangle$ denote orientational averaging.

In the low concentration approximation, the optical densities $D_{\|}$and $D_{\perp}$ are proportional to the corresponding components of the tensor (4),

$$
\begin{gathered}
D_{\|} \propto \rho_{\mathrm{A}}\left(\sigma_{\mathrm{av}}+2 \Delta \sigma S_{x x}^{(\mathrm{A})}\right) / 3, \\
D_{\perp} \propto \rho_{\mathrm{A}}\left(\sigma_{\mathrm{av}}+2 \Delta \sigma S_{y y}^{(\mathrm{A})}\right) / 3,
\end{gathered}
$$

so that the average absorption coefficient $D_{\text {av }}$ is given by

$$
D_{\mathrm{av}}=D_{\|}+2 D_{\perp} \propto \rho_{\mathrm{A}}\left[\sigma_{\mathrm{av}}+2 / 3 \Delta \sigma\left(S_{y y}^{(\mathrm{A})}-S_{z z}^{(\mathrm{A})}\right)\right] .
$$

When the absorption coefficient $D_{\text {av }}$ does not depend on the irradiation dose (and, thus, on the order parameter), from the expression (8) we may conclude that the anisotropy of the azo-dye film is uniaxial and $S_{y y}^{(\mathrm{A})}=S_{z z}^{(\mathrm{A})}=-S_{x x}^{(\mathrm{A})} / 2 \equiv-S_{A} / 2$. In this case we have

$$
\mathbf{S}_{\mathrm{A}}=S_{\mathrm{A}}(3 \hat{\mathbf{x}} \otimes \hat{\mathbf{x}}-\mathbf{I}) / 2, \quad R=\frac{\Delta \sigma}{\sigma_{\mathrm{av}}} S_{\mathrm{A}} .
$$


As is seen from Eq. (9), the dichroic ratio equals the order parameter only in the limiting case where absorption of waves propagating along the long molecular axis is negligibly small and $\sigma_{\perp} \rightarrow 0$.

\section{B. Anisotropic part of surface energy in the mean field approximation}

In the previous section it was shown that the light induced ordering of azo-dye molecules can be described by the order parameter (9) which is expected to affect the surface free energy at the nematic-substrate interface. So, in this section, the order parameter dependent part of the surface energy will be our primary concern.

In the case of a flat structureless substrate, the expression for the surface energy was originally obtained by Sen and Sullivan in Ref. [29]. Subsequently, similar results have been derived by using the mean field approximation [30] and the density functional theory [33-35].

Similarly to Ref. [30], we adopt the mean field approach and use the Fowler approximation for the one-particle distribution functions

$$
\rho_{\mathrm{N}}(\mathbf{r}, \hat{\mathbf{u}})=H(z) \rho_{\mathrm{N}}(z, \hat{\mathbf{u}}), \quad \rho_{\mathrm{A}}(\mathbf{r}, \hat{\mathbf{u}})=H(-z) \rho_{\mathrm{A}}(z, \hat{\mathbf{u}}),
$$

where $H(z)$ is the Heaviside step function which equals unity when $z$ is positive and vanishes otherwise.

Applying the mean field theory [30] gives the Landau-de Gennes surface free energy as an excess Helmholtz free energy per unit area that depends on two pair intermolecular potentials: (a) the potential of interaction between NLC molecules, $U_{\mathrm{N}-\mathrm{N}}\left(\mathbf{r}_{12}, \hat{\mathbf{u}}_{1}, \hat{\mathbf{u}}_{2}\right)$; and (b) the potential of interaction between NLC and azo-dye molecules, $U_{\mathrm{A}-\mathrm{N}}\left(\mathbf{r}_{12}, \hat{\mathbf{u}}_{1}, \hat{\mathbf{u}}_{2}\right)$, where $\mathbf{r}_{12}=\mathbf{r}_{1}-\mathbf{r}_{2}$ is the vector of intermolecular separation and $\hat{\mathbf{u}}_{i}$ is the orientation coordinates of the interacting molecules. The resulting expression is given by

$$
\begin{aligned}
& \Delta F / A= \int_{-\infty}^{0} d z_{1} \int_{0}^{\infty} d z_{2} \int d \hat{\mathbf{u}}_{1} d \hat{\mathbf{u}}_{2} \\
& \times\left[\rho_{\mathrm{A}}\left(z_{1}, \hat{\mathbf{u}}_{1}\right) V_{A}\left(z_{12}, \hat{\mathbf{u}}_{1}, \hat{\mathbf{u}}_{2}\right) \rho_{\mathrm{N}}\left(z_{2}, \hat{\mathbf{u}}_{2}\right)\right. \\
&\left.-\frac{1}{2} \rho_{\mathrm{N}}\left(z_{1}, \hat{\mathbf{u}}_{1}\right) V_{\mathrm{N}}\left(z_{12}, \hat{\mathbf{u}}_{1}, \hat{\mathbf{u}}_{2}\right) \rho_{\mathrm{N}}\left(z_{2}, \hat{\mathbf{u}}_{2}\right)\right], \\
& V_{\alpha}\left(z_{12}, \hat{\mathbf{u}}_{1}, \hat{\mathbf{u}}_{2}\right)=\int_{A} U_{\alpha-\mathrm{N}}\left(\mathbf{r}_{12}, \hat{\mathbf{u}}_{1}, \hat{\mathbf{u}}_{2}\right) d x_{12} d y_{12},
\end{aligned}
$$

where $A$ is the area of the substrate and $V_{\alpha}$ is the potential averaged over in-plane coordinates.

It should be noted that the potentials $U_{\mathrm{N}-\mathrm{N}}$ and $U_{\mathrm{A}-\mathrm{N}}$ actually represent the perturbative part of interaction that can be treated in the mean field approximation. They can be written in the form of an expansion over spherical harmonics given in Eq. (B1) of Appendix B. For our purposes, however, it is more convenient to use the tensorial representation for the averaged potentials $V_{\alpha}$ that was introduced in Ref. [36]. In Appendix B, the coefficients that enter this representation [see Eq. (B4)] are related to the coefficients $v_{j_{1} j_{2} j}(z)$ with $j_{i}$ $<4$, in the spherical harmonics expansion (B4). This relation is given by Eqs. (B9)-(B12).

Substituting the representation (B4) into Eq. (11) and assuming homogeneity of the contacting phases, we obtain the Landau-de Gennes expression for the surface free energy in the final form

$$
\begin{gathered}
f_{S}\left(\mathbf{S}_{\mathrm{N}}, \mathbf{S}_{\mathrm{A}}\right)=f_{\mathrm{N}}\left(\mathbf{S}_{\mathrm{N}}\right)++f_{\mathrm{A}}\left(\mathbf{S}_{\mathrm{N}}, \mathbf{S}_{\mathrm{A}}\right), \\
f_{\mathrm{N}}\left(\mathbf{S}_{\mathrm{N}}\right)=c_{0} \hat{\mathbf{z}} \cdot \mathbf{S}_{\mathrm{N}} \cdot \hat{\mathbf{z}}+c_{\mathrm{N}}^{(1)} \operatorname{Tr}\left(\mathbf{S}_{\mathrm{N}}^{2}\right)+c_{\mathrm{N}}^{(2)} \hat{\mathbf{z}} \cdot \mathbf{S}_{\mathrm{N}}^{2} \cdot \hat{\mathbf{z}} \\
+c_{\mathrm{N}}^{(3)}\left(\hat{\mathbf{z}} \cdot \mathbf{S}_{\mathrm{N}} \cdot \hat{\mathbf{z}}\right)^{2}, \\
f_{\mathrm{A}}\left(\mathbf{S}_{\mathrm{N}}, \mathbf{S}_{\mathrm{A}}\right)=c_{\mathrm{A}}^{(1)} \operatorname{Tr}\left(\mathbf{S}_{\mathrm{N}} \mathbf{S}_{\mathrm{A}}\right)+c_{\mathrm{A}}^{(2)} \hat{\mathbf{z}} \cdot \mathbf{S}_{\mathrm{N}} \mathbf{S}_{\mathrm{A}} \cdot \hat{\mathbf{z}} \\
\quad+c_{\mathrm{A}}^{(3)}\left(\hat{\mathbf{z}} \cdot \mathbf{S}_{\mathrm{N}} \cdot \hat{\mathbf{z}}\right) \cdot\left(\hat{\mathbf{z}} \cdot \mathbf{S}_{\mathrm{A}} \cdot \hat{\mathbf{z}}\right),
\end{gathered}
$$

where the coefficients are given by

$$
\begin{gathered}
c_{0}=b_{\mathrm{A}}^{(0)}-b_{\mathrm{N}}^{(0)}, \\
c_{\mathrm{A}}^{(i)}=b_{\mathrm{A}}^{(i)}, \quad c_{\mathrm{N}}^{(i)}=-b_{\mathrm{N}}^{(i)} / 2, \\
b_{\alpha}^{(i)}=\rho_{\alpha} \rho_{\mathrm{N}} \int_{0}^{\infty} z \beta_{\alpha}^{(i)}(z) d z .
\end{gathered}
$$

$\beta_{\alpha}^{(i)}(z)$ denote the coefficients in the representation (B4) for the potential (12).

Equations (13)-(15) can be viewed as a generalization of the expression by Sen and Sullivan [29] supplemented with the term $f_{\mathrm{A}}\left(\mathbf{S}_{\mathrm{N}}, \mathbf{S}_{\mathrm{A}}\right)$ resulting from the interaction between the NLC and azo-dye molecules. Note that this result can also be derived by constructing invariants from the order parameter tensors $\mathbf{S}_{\alpha}$ and the normal to the substrate $\hat{\mathbf{z}}$. In this case, the surface of the azo-dye aligning film is treated phenomenologically as a bounding surface which, in addition to the normal $\hat{\mathbf{z}}$, is characterized by the order parameter $\mathbf{S}_{\mathrm{A}}$.

\section{Bare anchoring energy}

Separating out the director dependent part of the surface free energy requires that the order parameters of azo-dye and NLC molecules be substituted into Eqs. (13)-(15). Since the order parameter at the surface may differ from its value in the bulk, we generalize the expression for the azo-dye order parameter (9) as follows:

$$
\left.2 \mathbf{S}_{\mathrm{A}}\right|_{z=0}=S_{\mathrm{A}}(3 \hat{\mathbf{x}} \otimes \hat{\mathbf{x}}-\mathbf{I})+P_{\mathrm{A}}(\hat{\mathbf{z}} \otimes \hat{\mathbf{z}}-\hat{\mathbf{y}} \otimes \hat{\mathbf{y}}) .
$$

Similarly, for the NLC order parameter tensor at $z=0$, from Eq. (A15) we have

$$
\begin{aligned}
\left.2 \mathbf{S}_{\mathrm{N}}\right|_{z=0} & =S(3 \hat{\mathbf{n}} \otimes \hat{\mathbf{n}}-\mathbf{I})+P(\hat{\mathbf{m}} \otimes \hat{\mathbf{m}}-\hat{\boldsymbol{l}} \otimes \hat{\boldsymbol{l}}) \\
& =(3 S+P) \hat{\mathbf{n}} \otimes \hat{\mathbf{n}}+2 P \hat{\mathbf{m}} \otimes \hat{\mathbf{m}}-(S+P) \mathbf{I},
\end{aligned}
$$

where $\hat{\mathbf{n}}=(\sin \Theta \cos \Phi, \sin \Theta \sin \Phi, \cos \Theta)$ is the NLC director, $\quad \hat{\mathbf{n}} \perp \hat{\mathbf{m}}=\cos \gamma \mathbf{e}_{x}(\hat{\mathbf{n}})-\sin \gamma \mathbf{e}_{y}(\hat{\mathbf{n}}), \quad \mathbf{e}_{x}(\hat{\mathbf{n}})=(\cos \Theta$ $\cos \Phi, \cos \Theta \sin \Phi,-\sin \Theta), \mathbf{e}_{y}(\hat{\mathbf{n}})=(-\sin \Phi, \cos \Phi, 0)$, and $\hat{\boldsymbol{l}}=\hat{\mathbf{n}} \times \hat{\mathbf{m}}$. 
Equations (19) and (20) suggest that the order parameters of azo-dye and NLC molecules though both uniaxial in the bulk can be biaxial at the surface. In addition, the scalar order parameters $S_{\mathrm{A}}$ and $S$ at the surface may also deviate from their values in the bulk.

The surface free energy can now be expressed as a sum of two contributions,

$$
f_{S}\left(\mathbf{S}, \mathbf{S}_{\mathrm{A}}\right)=W(\hat{\mathbf{n}}, \hat{\mathbf{m}})+f_{\text {scal }},
$$

where $W(\hat{\mathbf{n}}, \hat{\mathbf{m}})$ is the orientation dependent part of the surface energy. This part can be calculated by substituting the order parameters (19) and (20) into the surface energy (13) to yield the expression for the bare anchoring energy,

$$
\begin{aligned}
W(\hat{\mathbf{n}}, \hat{\mathbf{m}})= & N_{z}(\hat{\mathbf{n}} \cdot \hat{\mathbf{z}})^{2}+M_{z}(\hat{\mathbf{m}} \cdot \hat{\mathbf{z}})^{2}+N_{x}(\hat{\mathbf{n}} \cdot \hat{\mathbf{x}})^{2}+M_{x}(\hat{\mathbf{m}} \cdot \hat{\mathbf{x}})^{2} \\
& +c_{\mathrm{N}}^{(3)} / 4\left[(3 S+P)(\hat{\mathbf{n}} \cdot \hat{\mathbf{z}})^{2}+2 P(\hat{\mathbf{m}} \cdot \hat{\mathbf{z}})^{2}\right]^{2}
\end{aligned}
$$

with the coefficients defined by the relations

$$
\begin{gathered}
4 N_{z}=(3 S+P)\left[q+c_{\mathrm{N}}^{(2)}(S-P)\right], \\
4 N_{x}=c_{\mathrm{A}}^{(1)}\left(3 S_{\mathrm{A}}+P_{\mathrm{A}}\right)(3 S+P), \\
2 M_{z}=P\left(q-2 c_{\mathrm{N}}^{(2)} S\right), \quad 2 M_{x}=c_{\mathrm{A}}^{(1)}\left(3 S_{\mathrm{A}}+P_{\mathrm{A}}\right) P, \\
q \equiv 2 c_{0}-\left(c_{\mathrm{A}}^{(2)}+c_{\mathrm{A}}^{(3)}\right)\left(S_{\mathrm{A}}-P_{\mathrm{A}}\right)+2 c_{\mathrm{A}}^{(1)} P_{\mathrm{A}}-2 c_{\mathrm{N}}^{(3)}(S+P) .
\end{gathered}
$$

The second term on the right hand side of Eq. (21),

$$
\begin{aligned}
4 f_{\text {scal }}= & -\left[2 c_{0}-\left(S_{\mathrm{A}}-P_{\mathrm{A}}\right)\left(c_{\mathrm{A}}^{(2)}+c_{\mathrm{A}}^{(3)}\right)+3 c_{\mathrm{A}}^{(1)}\left(S_{\mathrm{A}}+P_{\mathrm{A}}\right)\right] \\
& \times(S+P)+\left(c_{\mathrm{N}}^{(2)}+c_{\mathrm{N}}^{(3)}\right)(S+P)^{2}+2 c_{\mathrm{N}}^{(1)}\left(3 S^{2}+P^{2}\right),
\end{aligned}
$$

is a quadratic function of the NLC scalar order parameter $S$ and the biaxiality $P$. From Eq. (27), like the anchoring energy (22), it depends linearly on the azo-dye parameters $S_{\mathrm{A}}$ and $P_{\mathrm{A}}$.

For the anchoring energy (22), we consider the simplest case which occurs when the surface induced NLC biaxiality $P$ is negligibly small and the quadrupolar term $v_{224}$ in the expansion of the intermolecular potential $V_{\mathrm{N}}$ can be ignored. Under these circumstances, setting $P=c_{\mathrm{N}}^{(3)}=0$ and $M_{z}=M_{x}$ $=0$, we arrive at the simplified formula for the anchoring energy

$$
W(\hat{\mathbf{n}})=N_{z}(\hat{\mathbf{n}} \cdot \hat{\mathbf{z}})^{2}+N_{x}(\hat{\mathbf{n}} \cdot \hat{\mathbf{x}})^{2}
$$

which agrees with the expression for the anchoring energy recently proposed in Refs. [37-39].

From Eq. (28) it is clear that the easy axis is directed along the $y$ axis, $\mathbf{e}_{s}=\hat{\mathbf{y}}$, only if the coefficients $N_{z}$ and $N_{z}$ are both positive. In this case the polar and the azimuthal anchoring strengths, $W_{\theta}$ and $W_{\phi}$, are given by

$$
W_{\theta}=2 N_{z}, \quad W_{\phi}=2 N_{x} .
$$

From Eq. (24) we immediately deduce a more explicit expression for the azimuthal anchoring strength,

$$
W_{\phi}=w_{\phi}\left(\left.S_{y y}^{(\mathrm{A})}\right|_{z=0}-\left.S_{x x}^{(\mathrm{A})}\right|_{z=0}\right) \text {, }
$$

$$
2 w_{\phi}=-\left.3 c_{\mathrm{A}}^{(1)} S\right|_{z=0},
$$

where the notations indicate the plane $z=0$ as the surface separating the phases.

Similarly, Eq. (23) gives the polar anchoring strength in the explicit form

$$
\begin{gathered}
W_{\theta}=w_{\theta}^{(0)}+\left.w_{\theta}^{(1)} S_{z z}^{(\mathrm{A})}\right|_{z=0}-w_{\phi}\left(\left.S_{z z}^{(\mathrm{A})}\right|_{z=0}-\left.S_{y y}^{(\mathrm{A})}\right|_{z=0}\right), \\
2 w_{\theta}^{(1)}=\left.3\left(c_{\mathrm{A}}^{(2)}+c_{\mathrm{A}}^{(3)}\right) S\right|_{z=0} .
\end{gathered}
$$

The formulas (30)-(33) will be subsequently used in Sec. IV to interpret the experimental data. At this stage, it is worth noting that, for the order parameter (9), the relations (31) and (33) provide the inequalities

$$
c_{\mathrm{A}}^{(1)}<0, \quad c_{\mathrm{A}}^{(2)}+c_{\mathrm{A}}^{(3)}>0
$$

as conditions for the anchoring strengths to increase linearly as the scalar order parameter $S_{\mathrm{A}}$ decreases.

\section{Model of spatially varying order parameter}

As was pointed out at the beginning of the previous section, the surface order parameter tensor of an azo-dye film (19) may differ from the bulk order parameter of the film (9). The latter, according to the experimental results presented in the subsequent Sec. III, is uniaxially anisotropic with an inplane anisotropy axis that is normal to the polarization vector of the activating uv light. In addition, the light induced scalar order parameter, which is proportional to the dichroic ratio (3), turns out to be negative, $S_{\mathrm{A}}^{(b)}<0$.

On the other hand, assuming that the anisotropic part of the surface energy can be taken in the general form by Sen and Sullivan [29], the boundary conditions may favor either homeotropic or planar alignment of the azo-dye molecules, thus counteracting the action of light. So it can be expected that the effects caused by interplay between the light induced and the surface ordering are of importance in explaining the order parameter dependencies of the polar and azimuthal anchoring energies.

In this section we discuss these effects on the basis of a simple phenomenological model formulated by using the polar representation (A21) for the azo-dye order parameter. The latter can be conveniently rewritten in the form

$$
S_{\mathrm{A}}=-s_{\mathrm{A}} \cos \psi, \quad P_{\mathrm{A}}=-\sqrt{3} s_{\mathrm{A}} \sin \psi,
$$

where the angle $\psi$ is shifted by $\pi$ so as to have the angle $\psi$ vanishing in the bulk.

In what follows we shall assume that, similarly to nematic liquid crystals [40-43], the amplitude $s_{\mathrm{A}}$ varies in space much more slowly than the angle $\psi$. So, in our model, the amplitude will be fixed at its bulk value, $s_{\mathrm{A}}=\left|S_{\mathrm{A}}^{(b)}\right|$, and we consider the limiting case of thick films in which the characteristic length of spatial variations of the angle $\psi$ is much shorter than the film thickness. In this case the film can be regarded as a semi-infinite sample filling the upper half space, $z \geqslant 0$.

Technically, our task will be to find the spatially varying angle $\psi$ as a function of $z$ that minimizes the excess free 
energy per unit area, $F_{\mathrm{A}}$, taken in the following nematiclike form:

$$
\begin{aligned}
F_{\mathrm{A}}= & \int_{0}^{\infty}\left\{L s_{\mathrm{A}}^{2}\left(\partial_{z} \psi\right)^{2}+B s_{\mathrm{A}}^{3}[1-\cos (3 \psi)]\right\} d z \\
& +G_{1} s_{\mathrm{A}} \cos \left(\psi_{0}+\pi / 3\right)+G_{2} s_{\mathrm{A}}^{2} \cos ^{2}\left(\psi_{0}+\pi / 3\right),
\end{aligned}
$$

where and $s_{\mathrm{A}} \cos \left(\psi_{0}+\pi / 3\right)=\left.\hat{\mathbf{z}} \cdot \mathbf{S}_{\mathrm{A}} \cdot \hat{\mathbf{z}}\right|_{z=0}$. The first part of the excess free energy (36) is of integral form with the integrand describing the energy costs for deviations of $\psi$ from the equilibrium value $\psi=0$. The gradient term of the energy density is taken to be proportional to $\left(\partial_{z} \mathbf{S}_{\mathrm{A}}\right)^{2}$, whereas the other term gives an increase in energy caused by spatially uniform changes in $\psi$. This term is written as a linear function of the angle dependent invariant (A19) $4 \operatorname{Tr}\left(\mathbf{S}_{\mathrm{A}}^{3}\right)=3 s_{\mathrm{A}}^{3} \cos (3 \psi)$.

For the order parameter (19), the surface part of the energy (36) can be represented by a quadratic polynomial of $\hat{\mathbf{z}} \cdot \mathbf{S}_{\mathrm{A}} \cdot \hat{\mathbf{z}}$. By contrast with the elastic constant $L$ and the coefficient $B$, the surface coupling constants $G_{1}$ and $G_{2}$ can generally be negative leading to different boundary conditions. For example, if $G_{2}=0$, minimizing the surface term requires the $z$ component of the order parameter, $\hat{\mathbf{z}} \cdot \mathbf{S}_{\mathrm{A}} \cdot \hat{\mathbf{z}}$ $=S_{z z}^{(\mathrm{A})}$, to attain its maximal (minimal) value at the surface provided the coefficient $G_{1}$ is negative (positive). These can be referred to as the homeotropic (planar) boundary conditions.

The Euler-Lagrange equation for the free energy functional (36) can be readily solved to yield the relation

$$
\tan (3 \psi / 4)=\tan \left(3 \psi_{0} / 4\right) \exp (-z / \xi),
$$

where $9 \xi^{2}=2 L\left(B s_{\mathrm{A}}\right)^{-1}$. This relation can now be substituted into Eq. (36) to derive the free energy as a function of the angle $\psi_{0}$. The result is

$$
\begin{aligned}
F_{\mathrm{A}} / h \equiv & \tilde{f}_{\mathrm{A}}\left(\psi_{0}\right)=s_{\mathrm{A}}^{5 / 2} \sin ^{2}\left(3 \psi_{0} / 4\right)+g_{1} s_{\mathrm{A}} \cos \left(\psi_{0}+\pi / 3\right) \\
& +g_{2} s_{\mathrm{A}}^{2} \cos ^{2}\left(\psi_{0}+\pi / 3\right),
\end{aligned}
$$

where $h=4(2 B L)^{1 / 2} / 3$ and $g_{i}=G_{i} / h$. The angle $\psi$ at the surface then can be found as the value of $\psi_{0}$ that minimizes the function (38) on the interval ranging from $-2 \pi / 3$ to $2 \pi / 3$.

Qualitatively, the dependence of $\psi_{0}$ on the coupling constant $g_{1}$ can be analyzed using elementary methods. For $g_{2}$ $\geqslant 0$, we find that the angle $\psi_{0}$ is localized within different intervals depending on the value of $g_{1}$. These are given by

$$
\begin{gathered}
-\pi / 3<\psi_{0} \leqslant 0, \quad g_{1} \leqslant g_{c}^{(1)}=-g_{2} s_{\mathrm{A}}, \\
0<\psi_{0} \leqslant \pi / 3, \quad g_{c}^{(1)}<g_{1} \leqslant g_{c}^{(2)}=g_{2} s_{\mathrm{A}}+\sqrt{3} / 2 s_{\mathrm{A}}^{3 / 2}, \\
\pi / 3<\psi_{0} \leqslant 2 \pi / 3, \quad g_{c}^{(2)}<g_{1} \leqslant g_{c}^{(3)}=2 g_{2} s_{\mathrm{A}}+9 / 8 s_{\mathrm{A}}^{3 / 2}, \\
\psi_{0}=2 \pi / 3, \quad g_{1}>g_{c}^{(3)} .
\end{gathered}
$$

The end points of the intervals in Eq. (39), $\psi_{0}=k \pi / 3$ with $-1 \leqslant k \leqslant 2$, represent the uniaxially anisotropic structures at the surface,

$$
\mathbf{S}_{\mathrm{A}}(0)=s_{\mathrm{A}}(3 \hat{\mathbf{z}} \otimes \hat{\mathbf{z}}-\mathbf{I}) / 2, \quad \psi_{0}=-\pi / 3,
$$

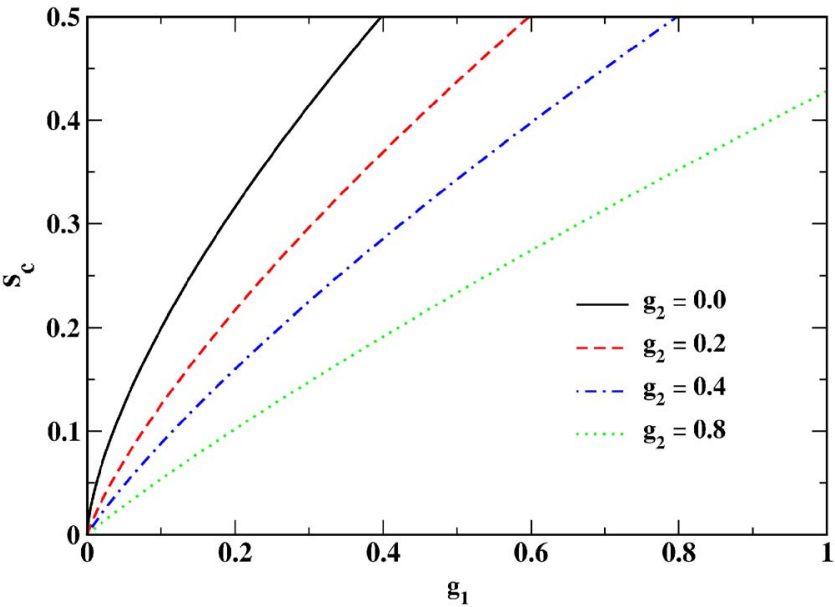

FIG. 1. (Color online) Critical order parameter $s_{c}$ as a function of the coupling constant $g_{1}$ at various values of the coefficient $g_{2}$.

$$
\begin{array}{cc}
\mathbf{S}_{\mathrm{A}}(0)=-s_{\mathrm{A}}(3 \hat{\mathbf{x}} \otimes \hat{\mathbf{x}}-\mathbf{I}) / 2, & \psi_{0}=0, \\
\mathbf{S}_{\mathrm{A}}(0)=s_{\mathrm{A}}(3 \hat{\mathbf{y}} \otimes \hat{\mathbf{y}}-\mathbf{I}) / 2, & \psi_{0}=\pi / 3, \\
\mathbf{S}_{\mathrm{A}}(0)=-s_{\mathrm{A}}(3 \hat{\mathbf{z}} \otimes \hat{\mathbf{z}}-\mathbf{I}) / 2, & \psi_{0}=2 \pi / 3 .
\end{array}
$$

From Eqs. (40) and (42) it is seen that, for the angles $\psi_{0}$ $=-\pi / 3$ and $\pi / 3$, surface alignment will be homeotropic and homogeneous (monostable planar), respectively. The structure (41) coincides with uniaxial ordering in the bulk (9) and the surface order parameter tensor (43) corresponds to planar (random in-plane) alignment.

According to Eq. (39), the case of planar alignment occurs only if the coupling constant $g_{1}$ is positive and the bulk order parameter $s_{\mathrm{A}}$ is below its critical value $s_{c}$ defined by the relation

$$
g_{1}=2 g_{2} s_{c}+9 / 8 s_{c}^{3 / 2} .
$$

Figure 1 shows that the critical order parameter $s_{c}$ is an increasing function of $g_{1}$.

In Fig. 2(a) we have plotted the curves representing the components of the order parameter tensor at the surface in relation to the order parameter in the bulk to illustrate that destruction of the planar alignment takes place in a secondorder transition manner.

However, it should be stressed that our model becomes inapplicable in the immediate vicinity of $s_{c}$ where the $z$ dependence of the biaxiality parameter $P_{\mathrm{A}}$ critically slows down. Actually, as can be inferred from Fig. 3, the characteristic length of spatially varying biaxiality diverges logarithmically as $s_{\mathrm{A}}$ approaches $s_{c}$ from above. Under these circumstances, the assumption that the scale of the spatial variations is much shorter than the film thickness is no longer justified.

By contrast to the boundary conditions with $g_{1}>0$, there are no second-order transitions provided the coupling constant $g_{1}$ is negative. At sufficiently large values of $\left|g_{1}\right|$, the surface ordering remains nearly homeotropic. Otherwise, the surface order parameter changes smoothly with $s_{\mathrm{A}}$ toward the bulk order parameter (41). From Eq. (39), the difference 


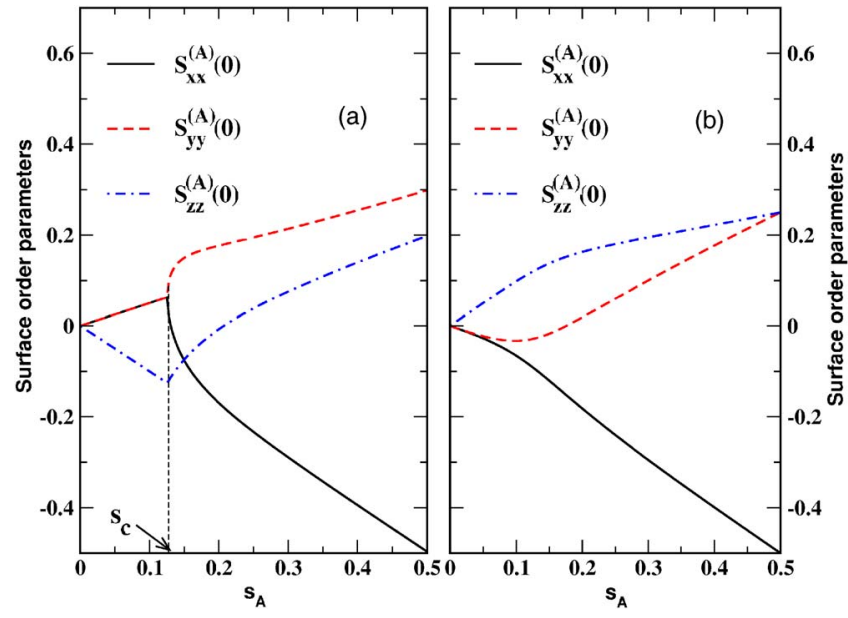

FIG. 2. (Color online) Components of the surface order parameter as a function of the bulk order parameter $s_{\mathrm{A}}$. Two cases are shown: (a) $g_{1}=0.05$ and $g_{2}=0.0$; (b) $g_{1}=-0.2$ and $g_{2}=0.4$.

between the order parameters vanishes when $g_{1}=-g_{2} s_{\mathrm{A}}$. The curves presented in Fig. 2(b) illustrate this point.

Leaving aside a detailed discussion of what happens when the coupling constant $g_{2}$ is negative, we just note that in this case the above discussed transition will generally be first order, leading to jumplike behavior of the order parameter at the surface.

\section{EXPERIMENT}

Now we pass on to describing the experimental procedure employed to obtain the data linking the anchoring energy strengths and the dichroic ratio as a measure of the photoinduced ordering. To this end, we carried out absorption spectra and anchoring energy measurements for azo-dye films irradiated at varying exposure time. Thus, we used samples prepared at different irradiation doses to measure the anchoring strengths and the dichroic ratio in relation to the dose. The data then can be recalculated to obtain the required anchoring energy vs dichroic ratio dependence.
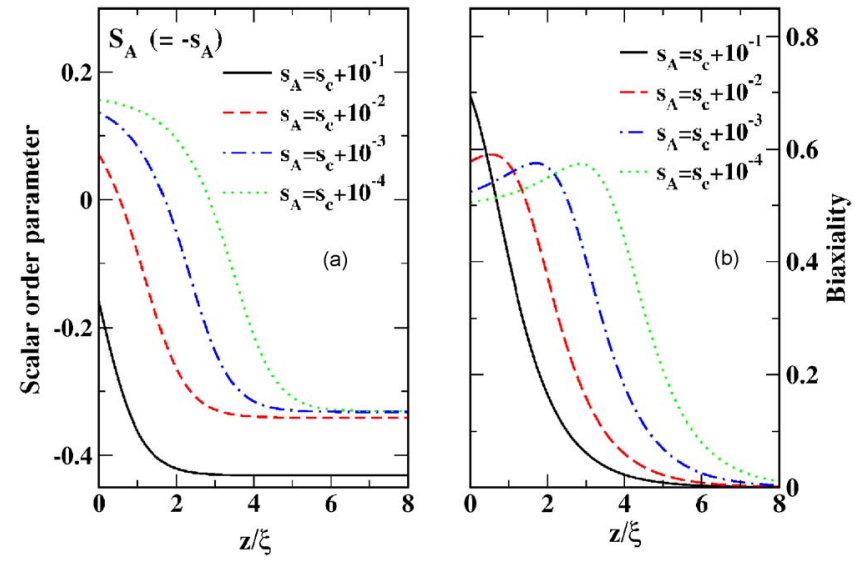

FIG. 3. (Color online) Spatial variations of (a) scalar order parameter and (b) biaxiality when the bulk order parameter $s_{\mathrm{A}}$ approaches its critical value $s_{c}$ at the planar boundary conditions with $g_{1}=0.05$ and $g_{2}=0.0$.

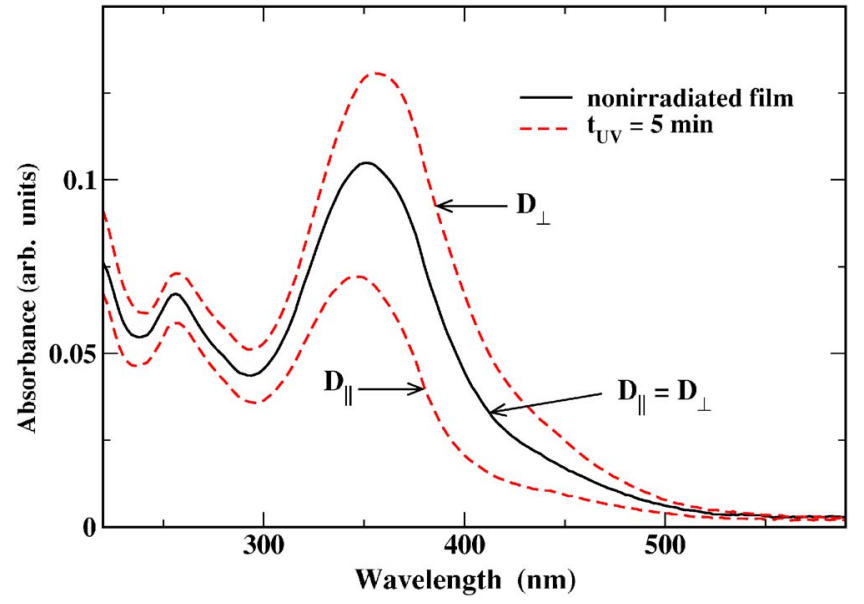

FIG. 4. (Color online) uv-visible absorption spectra of nonirradiated and irradiated azo-dye films.

\section{A. Sample preparation}

Following the method described in Ref. [21], the azobenzene sulfuric dye SD-1 was synthesized from the corresponding benzidinedisulfonic acid using azo coupling. The azo-dye compound SD-1 was mixed with the polymerizable azo-dye SDA-2 in the ratio $40 \%$ to $60 \%$. The mixture was dissolved in $N, N$-dimethylformamide and a heat initiator V-65 (from Wako Pure Chemical Industries, Ltd.) that was added in the ratio of 1:50 to SDA-2.

The solution was spin coated onto glass substrates with indium tin oxide electrodes at $800 \mathrm{rpm}$ for $5 \mathrm{~s}$ and, subsequently, at $3000 \mathrm{rpm}$ for $30 \mathrm{~s}$. The solvent was evaporated on a hot plate at $100{ }^{\circ} \mathrm{C}$ for $10 \mathrm{~min}$.

The surface of the coated film was illuminated with linearly polarized uv light using a super-high-pressure Hg lamp through an interference filter at the wavelength $365 \mathrm{~nm}$. The intensity of light irradiated on the film surface at varying time exposure was $2.7 \mathrm{~mW} / \mathrm{cm}^{2}$. After the photoaligning procedure, the SDA-2 films were polymerized by heating at $150{ }^{\circ} \mathrm{C}$ for $1 \mathrm{~h}$ in vacuum. In order to recover the quality of the photoalignment, degraded after the polymerization, the films were exposed to the uv light for 20 min regardless of the initial time exposure.

Two glass substrates with photoaligned films were assembled to form liquid crystal cells to measure the azimuthal and polar anchoring energy strengths. The cell thickness was 5 and $18 \mu \mathrm{m}$, respectively. Liquid crystal mixtures MLC6080 (from Merck) in an isotropic phase were injected into the cell by capillary action.

\section{B. Absorption spectra}

The uv-visible absorption spectra of the films were measured in the spectral range from 250 to $600 \mathrm{~nm}$ for normally incident probing light which is linearly polarized parallel (along the $x$ axis) and perpendicular (along the $y$ axis) to the polarization vector of the activating light.

For nonirradiated films, the curve shown in Fig. 4 as a solid line demonstrates that the absorption coefficient does not depend on the polarization state of the testing beam. By 


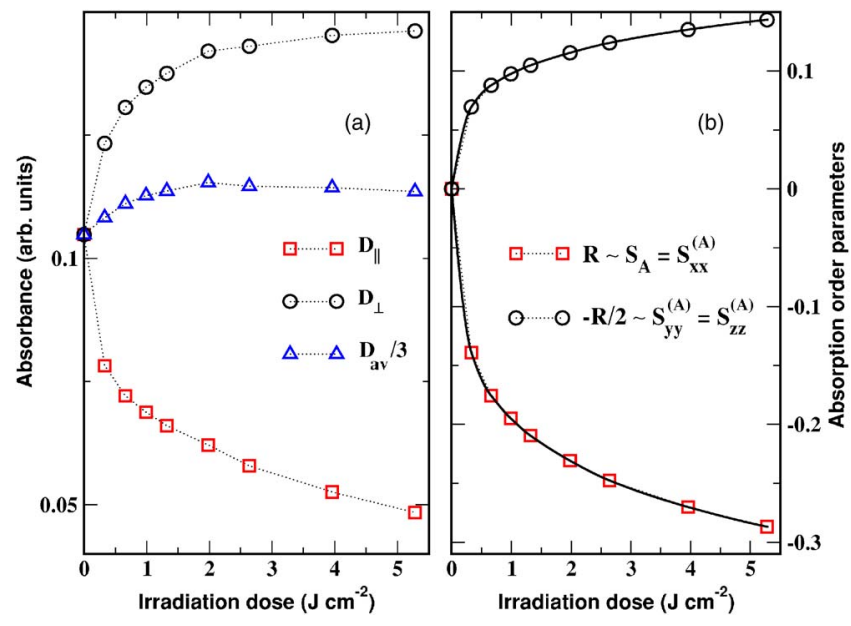

FIG. 5. (Color online) Dependence of (a) absorption coefficients and (b) absorption order parameters on uv irradiation dose. Solid lines give interpolation of the experimental data calculated using Akima splines.

contrast, as is illustrated in Fig. 4, the absorption coefficients $D_{\|}$and $D_{\perp}$ differ in the irradiated films, thus revealing the light induced absorption dichroism. This dichroism is mainly caused by the photoinduced angular redistribution of the azodye molecules.

By varying the exposure time the films were prepared at different irradiation doses and the optical density components $D_{\|}$and $D_{\perp}$ at the absorption maximum of azo-dyes $\left(\lambda_{m} \approx 350 \mathrm{~nm}\right)$ were estimated from the measured absorption spectra. The dichroic ratio then can be computed from the formula (3). The results for the absorption coefficients and the absorption order parameters, which are proportional to the dichroic ratio, are presented in Figs. 5(a) and 5(b), respectively.

\section{Anchoring energy strengths}

The azimuthal anchoring strength $W_{\phi}$ was measured in a twisted nematic cell using the torque balance method $[44,45]$. The azo-dye aligning film and a rubbed polyimide layer were used as confining substrates. The twist angle was $90^{\circ}$.

Measurements of the polar anchoring strength $W_{\theta}$ in antiparallel aligned cells were carried out using the high-voltage technique [22,46-48]. The experimental data for the azimuthal and polar anchoring strengths are plotted against the irradiation dose in Figs. 8(a) and 9(a), respectively. At low irradiation doses, when the exposure energy is below $1 \mathrm{~J} / \mathrm{cm}^{2}$, our experimental technique failed to provide accurate estimates for the anchoring strengths because of the poor quality of NLC alignment in the cells.

The experimentally measured dependence of the dichroic ratio on the irradiation dose can now be combined with the results for $W_{\phi}$ and $W_{\theta}$ so as to recalculate the anchoring energy strengths as functions of the dichroic ratio, $R$. The resulting data are shown in Figs. 8(b) and 9(b).

\section{RESULTS}

As is shown in Fig. 5(a) representing the absorption coefficients $D_{\|}$and $D_{\perp}$ measured in the film irradiated at vari-
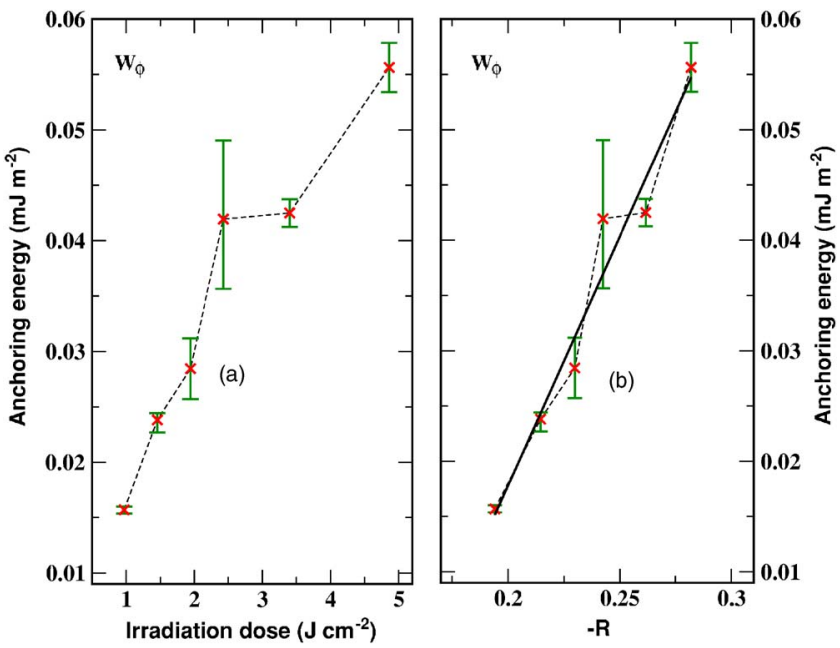

FIG. 6. (Color online) Azimuthal anchoring energy strength as a function of (a) uv irradiation dose and (b) dichroic ratio (3) which is proportional to the order parameter of azo-dye molecules, $S_{\mathrm{A}}$ [see Eq. (9)]. The solid line represents the result of linear fitting $W_{\phi} \approx$ $-w_{a}^{(0)}-w_{a}^{(1)} R$ with $w_{a}^{(0)}=0.07225 \mathrm{~mJ} / \mathrm{m}^{2}$ and $w_{a}^{(1)}=0.4503 \mathrm{~mJ} / \mathrm{m}^{2}$.

ous irradiation doses, within the limits of experimental error, the average absorption coefficient $D_{\text {av }}$ defined by the relation (8) remains unchanged at irradiation doses higher than $1 \mathrm{~J} / \mathrm{cm}^{2}$. So, from the discussion given at the end of Sec. II A we conclude that the azo-dye order parameter in the bulk of the film is uniaxial and is of the form given by Eq. (9). In Fig. 5(b), it is indicated that the components of the order parameter tensor are proportional to the dichroic ratio (3). Clearly, for $D_{\perp}>D_{\|}$and $\sigma_{\|}>\sigma_{\perp}$, the azo-dye order parameter $S_{\mathrm{A}}$ and $R$ are both negative.

The experimental results for the azimuthal and polar anchoring strengths measured in NLC cells with photoaligned azo-dye films used as aligning substrates are presented in Figs. 6(a) and 7(a), respectively. The films differ in the amount of photoinduced anisotropy which is controlled by varying exposure time and the anchoring strengths are plotted in relation to the irradiation dose.

However, the fundamentally important characteristic describing degree of the photoinduced anisotropy is the azodye order parameter. So, in order to compare the experimental data and the theory, we need to relate the anchoring strengths and the dichroic ratio. Combining the anchoring energy data and the curve depicted in Fig. 5(b) gives the result shown in Figs. 6(b) and 7(b).

From the relations (30)-(33) the anchoring strengths depend linearly on the dichroic ratio provided the order parameters at the surface do not differ from their values in the bulk. The results of linear fitting of the experimental data are shown as solid straight lines in Figs. 6(b) and 7(b).

Referring to Fig. 6(b), the linear approximation for $W_{\phi}$ predicts that the azimuthal anchoring strength vanish at a certain nonzero value of the dichroic ratio $R \approx-0.16$. By contrast, from the formulas (30) and (31) the anchoring strength $W_{\phi}$ is proportional to $R$ and thus disappears only in the limit of weak photoinduced anisotropy where $R \rightarrow 0$. Assuming that this discrepancy can be attributed to the difference between the bulk and surface order parameters of the 


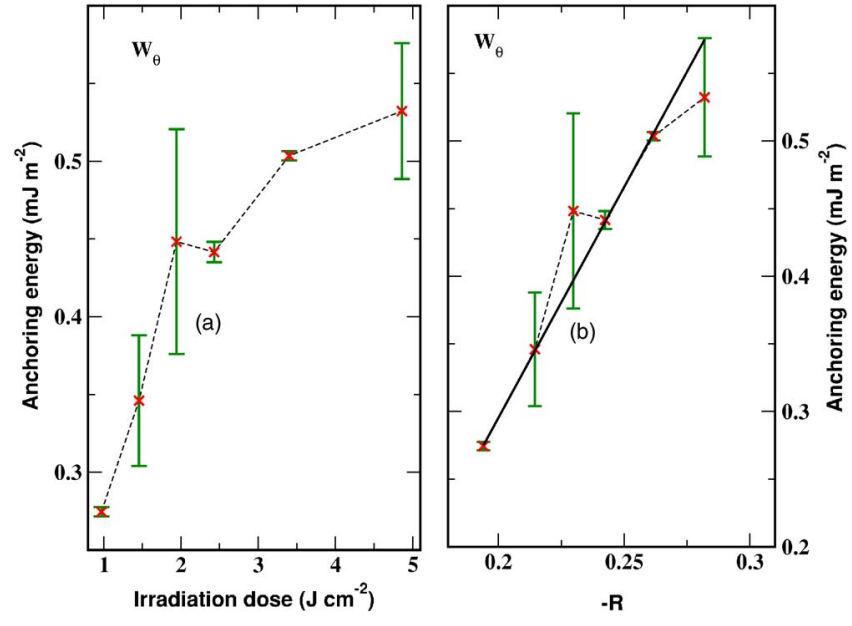

FIG. 7. (Color online) Polar anchoring energy strength as a function of (a) uv irradiation dose and (b) dichroic ratio (3) which is proportional to the order parameter of azo-dye molecules, $S_{\mathrm{A}}$ [see Eq. (9)]. The solid line represents the result of linear fitting $W_{\theta}$ $\approx-w_{p}^{(0)}-w_{p}^{(1)} R$ with $w_{p}^{(0)}=0.38714 \mathrm{~mJ} / \mathrm{m}^{2}$ and $w_{p}^{(1)}=3.4116$ $\mathrm{mJ} / \mathrm{m}^{2}$

azo-dye, we can apply the phenomenological model described in Sec. II D to interpret the experimental data.

In the angle-amplitude representation (35), the surface order parameters that enter the expression (30) are given by

$$
\left.S_{x x}^{(\mathrm{A})}\right|_{z=0}=-s_{\mathrm{A}} \cos \psi_{0},\left.\quad S_{y y}^{(\mathrm{A})}\right|_{z=0}=s_{\mathrm{A}} \cos \left(\psi_{0}-\pi / 3\right),
$$

where $-s_{\mathrm{A}}=S_{\mathrm{A}}=\left(\Delta \sigma / \sigma_{\mathrm{av}}\right) R$ is the scalar order parameter in the bulk of the azo-dye film [see Eq. (9)]. According to our model, $\psi_{0}$ is the angle that minimizes the energy (38).

So, the computational procedure involves two steps: (a) minimization of the energy (38) to find the angle $\psi_{0}$; and (b) using the relations (45) to compute the azimuthal anchoring energy (30). Following this procedure, we may calculate dependence of the anchoring strength $W_{\phi}$ on the photoinduced order parameter $s_{\mathrm{A}}$.

As it is discussed in Sec. II D, the results crucially depend on the boundary conditions that are determined by two coupling constants $g_{1}$ and $g_{2}$. At $g_{1} \geqslant 0$, the surface favors planar (random in-plane) alignment of the azo-dye molecules. In the opposite case of negative coupling constant $g_{1}$, the alignment is homeotropic.

In Fig. 8, we show the theoretical curves calculated for both planar and homeotropic boundary conditions. The corresponding numerical results for the polar anchoring strength are presented in Fig. 9.

The curve plotted in Fig. 8(a) indicates that, for planar boundary conditions, the azimuthal anchoring strength $W_{\phi}$ takes nonzero values and starts growing only if the azo-dye order parameter $s_{\mathrm{A}}$ exceeds its critical value $s_{c}$. Such threshold behavior is a consequence of the second-order transition discussed in Sec. II D. In contrast, as is shown in Fig. 8(b), $W_{\phi}$ is a smoothly increasing function of $s_{\mathrm{A}}$ when the boundary conditions favor the homeotropic alignment at the surface.

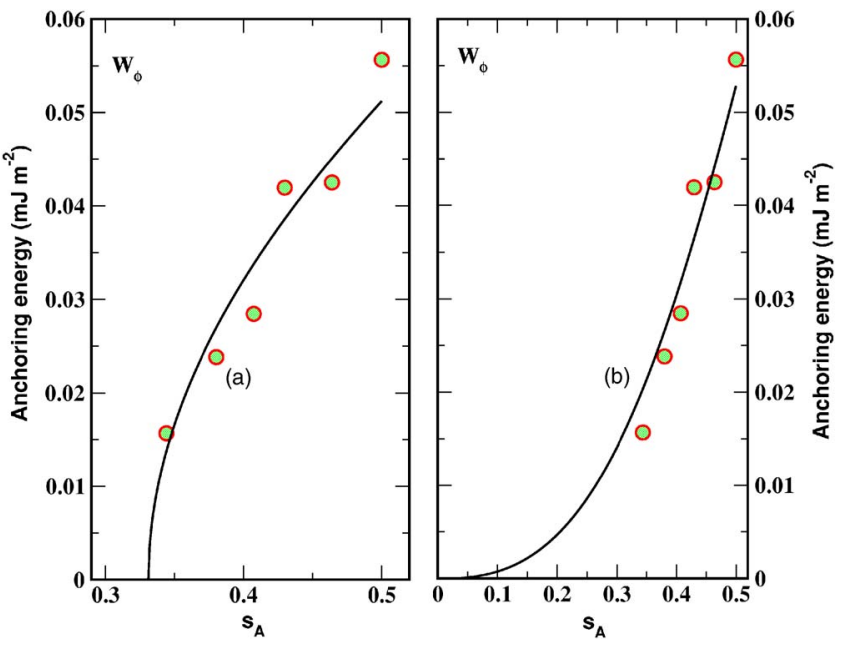

FIG. 8. (Color online) Azimuthal anchoring energy strength versus azo-dye order parameter. Solid lines represent theoretical curves computed from the formula (30) by minimizing the energy (38) for two different sets of the parameters: (a) $g_{1}=0.47, g_{2}=0.4, w_{\phi}$ $=0.067 \mathrm{~mJ} / \mathrm{m}^{2}$; (b) $g_{1}=-0.9, g_{2}=0.4, w_{\phi}=0.157 \mathrm{~mJ} / \mathrm{m}^{2}$.

At first glance, the curves representing the polar anchoring strength $W_{\theta}$ plotted against $s_{\mathrm{A}}$ in Figs. 9(a) and 9(b) do not show any noticeable differences. It should be stressed, however, that the planar boundary conditions prevent the polar anchoring energy from decaying to zero as the order parameter $s_{\mathrm{A}}$ decreases. From Fig. 9(b) it can be seen that this is no longer the case when the coupling constant $g_{1}$ becomes negative.

These results show that interplay between photoinduced ordering in the bulk of the azo-dye films and the preferred alignment of the azo-dye molecules at the surface may have a profound effect on the order parameter dependence of the
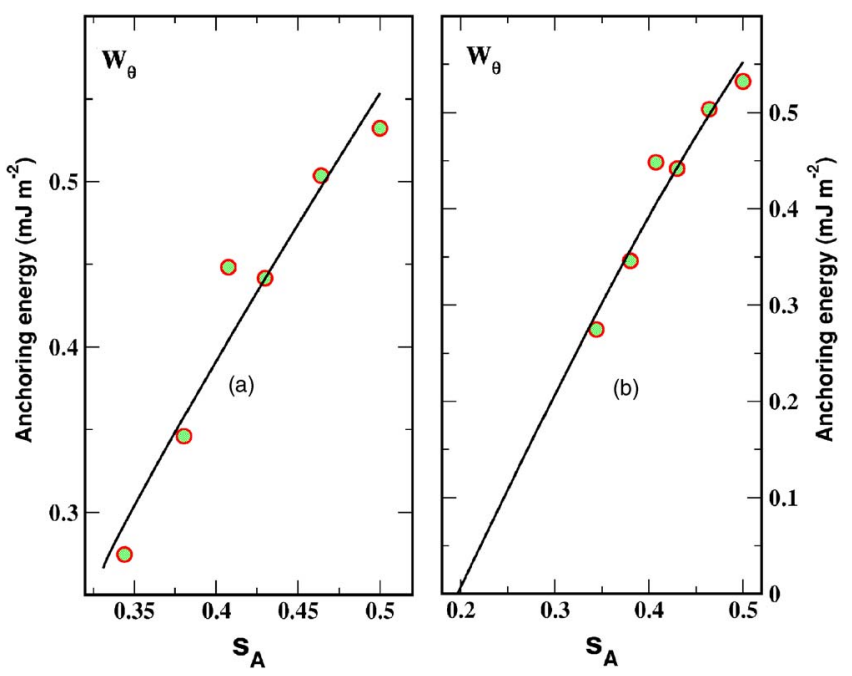

FIG. 9. (Color online) Polar anchoring energy strength versus azo-dye order parameter. Solid lines represent theoretical curves computed from the formula (32) by minimizing the energy (38) for two different sets of the parameters: (a) $g_{1}=0.47, g_{2}=0.4, w_{\theta}^{(0)}$ $=1.113 \mathrm{~mJ} / \mathrm{m}^{2}, \quad w_{\theta}^{(1)}=2.711 \mathrm{~mJ} / \mathrm{m}^{2} ; \quad$ (b) $g_{1}=-0.9, \quad g_{2}=0.4, \quad w_{\theta}^{(0)}$ $=-0.382 \mathrm{~mJ} / \mathrm{m}^{2}, w_{\theta}^{(1)}=2.247 \mathrm{~mJ} / \mathrm{m}^{2}$. 
anchoring energies. For the planar alignment with $g_{1}>0$, our model predicts that the surface ordering may change through the second-order transition as the photoinduced anisotropy increases. This transition bears close resemblance to the second-order transitions in nematic liquid crystals previously studied in Refs. [49,50].

Quantitatively, the polar anchoring energy $W_{\theta}$ appears to be an order of magnitude higher than the azimuthal energy $W_{\phi}$. From our estimates, the ratio of the coefficients $w_{\theta}^{(1)}$ and $w_{\phi}, w_{\theta}^{(1)} / w_{\phi}=-\left(c_{\mathrm{A}}^{(2)}+c_{\mathrm{A}}^{(3)}\right) / c_{\mathrm{A}}^{(1)}$, is likely to be well above 10 .

The coefficient $c_{\mathrm{A}}^{(1)}$ is negative and its sign is determined by the dominating contribution from the Maier-Saupe term $v_{220}$ of the spherical harmonics expansion for the azo-dyeNLC intermolecular potential $U_{\mathrm{A}-\mathrm{N}}$. From Eq. (B10) the absolute value of $c_{\mathrm{A}}^{(2)}$ can be significantly reduced when the quadrupole term $v_{224}$ and the harmonics $v_{222}$ are predominantly positive, so that $\int_{0}^{\infty} z v_{224}(z) d z>0$ and $\int_{0}^{\infty} z v_{222}(z) d z$. $>0$.

Under these circumstances, the condition (34), which requires the sum of $c_{\mathrm{A}}^{(2)}$ and $c_{\mathrm{A}}^{(3)}$ to be positive, can be satisfied only if the contribution of the quadrupole term to the sum $c_{\mathrm{A}}^{(2)}+c_{\mathrm{A}}^{(3)}$ is dominating. Thus, we may conclude that the quadrupole term is of vital importance for understanding the reasons behind the significant difference in magnitude between the photoinduced parts of the polar and azimuthal anchoring strengths.

\section{DISCUSSION AND CONCLUSIONS}

In this paper we have studied both theoretically and experimentally the effects of photoinduced ordering in azo-dye aligning films on anchoring energy strengths. These effects are governed by the dependence of the strengths on the azodye order parameter.

Our theoretical approach relies on the mean field theory [30] and provides general expressions for the Landau-de Gennes surface free energy (13) and the anchoring energy (22). The theoretical results for the azimuthal and polar anchoring energy strengths are obtained under certain simplifying assumptions and are used to interpret the experimental data relating the anchoring strengths and the dichroic ratio.

We found that linear fitting of the data for the azimuthal anchoring strength, though giving good results, predicts, in contradiction to the bare anchoring theory, the effect of vanishing anchoring which occurs at a certain nonzero value of the dichroic ratio (and thus the irradiation dose). By using a simple phenomenological model we have shown that this effect can be attributed to interplay between the light induced ordering in the bulk and the boundary conditions at the surface of the film which may counteract the action of light. Thus, the bulk and surface values of the azo-dye order parameter are generally different.

For planar boundary conditions that favor the random inplane alignment of the azo-dye molecules, our model predicts threshold behavior of the azimuthal anchoring strength that starts growing provided the bulk order parameter $s_{\mathrm{A}}$ exceeds its critical value. When the boundary conditions are homeotropic, this is no longer the case and the azimuthal strength smoothly increases with the dichroic ratio.

The results presented in Figs. 8 and 9 demonstrate that the theoretical curves calculated for both types of the boundary conditions can fit the experimental data equally well. There are, however, a number of differences concerning the polar anchoring strength. The results of linear fitting presented in Fig. 7(b) are less satisfactory than those for the azimuthal anchoring strength. This gives extra evidence for the above discussed breakdown of the bare anchoring theory. What is more important, in planar conditions, as opposed to the case of the homeotropic boundary conditions, the polar anchoring strength never equals zero. So, from the previously published results $[22,51]$, it can be concluded that the homeotropic boundary condition is unlikely to occur in the azo-dye films under consideration.

Our final remarks concern some of the assumptions made to simplify our theoretical analysis. Within the density functional formalism, the mean field approach, employed in Sec. II to deduce the expression for the anchoring energy, implies using the mean spherical approximation for the OrnsteinZernike direct correlation function [30,52]. A more sophisticated treatment would renormalize the perturbative part of the intermolecular potentials, but preserve the form of the bare anchoring energy (22). More importantly, surface adsorption phenomena cannot be taken into account without going beyond the Fowler approximation (10).

Our model presented in Sec. II D suggests that spatial variation of the azo-dye order parameter is the crucial factor responsible for discrepancy between the experimental results and the bare anchoring theory. The model relies on the square-gradient approximation and is formulated along a line of reasoning which is closely analogous to the Cahn wetting theory [53] (a more recent review can be found in Ref. [54]).

Although the results show that our assumptions are reasonable, a more comprehensive analysis would require a quantitatively accurate theory based on a self-consistent treatment of two order parameter tensors in the interfacial layer. This task still remains a challenge and we hope that our study will stimulate further progress in the field.

\section{ACKNOWLEDGMENTS}

This research was partially supported by RGC Grants No. HKUST6102/03E and No. HKUST6149/04E. A.D.K. is indebted to Professor T. J. Sluckin (University of Southampton, U.K.) for numerous useful conversations and acknowledges partial support from INTAS under Grant No. 03-515448. We are also grateful to Professor H.-S. Kwok for stimulating comments.

\section{APPENDIX A: IRREDUCIBLE TENSORS, ORDER PARAMETER, AND INVARIANTS}

In this appendix we introduce notations and definitions used throughout the paper. In addition, we express the order parameter in terms of irreducible tensors and deduce a number of algebraic relations simplifying the derivation of the tensorial form of the intermolecular potential given in Appendix B. 
The irreducible tensors $\mathbf{T}_{m}$ with the azimuthal number $m$ ranging from -2 to 2 can be defined as linear combinations of the following form [55]:

$$
\mathbf{T}_{m}=\sum_{\mu, \nu=-1}^{1} C_{\mu \nu m}^{112} \mathbf{e}_{\mu} \otimes \mathbf{e}_{\nu}
$$

where $C_{m_{1} m_{2} m}^{j_{1} j_{2} j}$ is the Clebsch-Gordan (Wigner) coefficient and $\mathbf{e}_{ \pm 1}=\mp(\hat{\mathbf{x}} \pm i \hat{\mathbf{z}}) / \sqrt{2}, \mathbf{e}_{0}=\hat{\mathbf{z}}$ are the vectors of the spherical basis $(\hat{\mathbf{x}}, \hat{\mathbf{y}}$, and $\hat{\mathbf{z}}$ are unit vectors directed along the corresponding coordinate axes). Substituting the values of the Wigner coefficient into Eq. (A1) gives the expressions for $\mathbf{T}_{m}$,

$$
\begin{gathered}
\mathbf{T}_{0}=\left(3 \mathbf{e}_{0} \otimes \mathbf{e}_{0}-\mathbf{I}\right) / \sqrt{6}, \\
\mathbf{T}_{ \pm 1}=\left(\mathbf{e}_{0} \otimes \mathbf{e}_{ \pm 1}+\mathbf{e}_{ \pm 1} \otimes \mathbf{e}_{0}\right) / \sqrt{2}, \\
\mathbf{T}_{ \pm 2}=\mathbf{e}_{ \pm 1} \otimes \mathbf{e}_{ \pm 1},
\end{gathered}
$$

so that it is not difficult to verify the validity of the orthogonality relation

$$
\operatorname{Tr}\left(\mathbf{T}_{m} \mathbf{T}_{-n}\right)=(-1)^{m} \delta_{m n}
$$

and the algebraic identities

$$
\hat{\mathbf{z}} \cdot \mathbf{T}_{m} \cdot \hat{\mathbf{z}}=\sqrt{2 / 3} \delta_{m 0}, \quad \hat{\mathbf{z}} \cdot \mathbf{T}_{m} \mathbf{T}_{-n} \cdot \hat{\mathbf{z}}=c_{|m|} \delta_{m n},
$$

where $c_{0}=2 / 3, c_{1}=-1 / 2$, and $c_{2}=0$.

Under the action of rotation the vectors of spherical basis transform as follows:

$$
\begin{gathered}
\mathbf{e}_{\mu} \rightarrow \mathbf{e}_{\mu}(\hat{\mathbf{u}})=\sum_{\nu=-1}^{1} D_{\nu \mu}^{1}(\hat{\mathbf{u}}) \mathbf{e}_{\nu}, \\
\mathbf{e}_{0}(\hat{\mathbf{u}})=\hat{\mathbf{u}}, \\
\mathbf{e}_{ \pm 1}(\hat{\mathbf{u}})=\mp\left[\mathbf{e}_{x}(\hat{\mathbf{u}}) \pm i \mathbf{e}_{y}(\hat{\mathbf{u}})\right] / \sqrt{2},
\end{gathered}
$$

where $D_{n m}^{j}(\hat{\mathbf{u}}) \equiv D_{n m}^{j}(\theta, \phi)$ is the Wigner $D$ function $[55,56]$; $\theta$ and $\phi$ are the Euler angles of the unit vector $\hat{\mathbf{u}} ; \mathbf{e}_{x}(\hat{\mathbf{u}})$ $=(\cos \theta \cos \phi, \cos \theta \sin \phi,-\sin \theta), \mathbf{e}_{y}(\hat{\mathbf{u}})=(-\sin \phi, \cos \phi, 0)$.

The definition (A1) implies that transformation properties of the tensors $\mathbf{T}_{m}$ under rotations are determined by the irreducible representation of the rotation group with $j=2$, where $j$ is the angular momentum number. So we have

$$
\mathbf{T}_{m} \rightarrow \mathbf{T}_{m}(\hat{\mathbf{u}})=\sum_{\mu, \nu=-1}^{1} C_{\mu \nu m}^{112} \mathbf{e}_{\mu}(\hat{\mathbf{u}}) \otimes \mathbf{e}_{\nu}(\hat{\mathbf{u}})=\sum_{k=-2}^{2} D_{k m}^{2}(\hat{\mathbf{u}}) \mathbf{T}_{k} .
$$

Equation (A10) can now be combined with the relations (A8) and (A2) to yield the expression for the order parameter tensor $\mathbf{Q}(\hat{\mathbf{u}})$ :

$$
\mathbf{Q}(\hat{\mathbf{u}})=\sqrt{3 / 2} \mathbf{T}_{0}(\hat{\mathbf{u}})=(3 \hat{\mathbf{u}} \otimes \hat{\mathbf{u}}-\mathbf{I}) / 2,
$$

where the unit vector $\hat{\mathbf{u}}$ is directed along the long molecular axis.

The director $\hat{\mathbf{n}}$ is defined as an eigenvector of the orientationally averaged order parameter tensor,

$$
\langle\mathbf{Q}(\hat{\mathbf{u}})\rangle_{\hat{\mathbf{u}}}=\sqrt{3 / 2} \sum_{k=-2}^{2}\left\langle D_{k 0}^{2}\left(\phi^{\prime}, \theta^{\prime}\right)\right\rangle \mathbf{T}_{k}(\hat{\mathbf{n}})
$$

where $\theta^{\prime}$ and $\phi^{\prime}$ are the Euler angles of the vector $\hat{\mathbf{u}}$ related to the basis vectors $\mathbf{e}_{i}(\hat{\mathbf{n}})$.

Since $\hat{\mathbf{n}}$ is the director, the averages $\left\langle D_{ \pm 10}^{2}\left(\phi^{\prime}, \theta^{\prime}\right)\right\rangle_{\phi^{\prime}, \theta^{\prime}}$ vanish. Other averages

$$
\begin{gathered}
\left\langle D_{00}^{2}\left(\phi^{\prime}, \theta^{\prime}\right)\right\rangle=\sqrt{2 / 3} S \\
\left\langle D_{ \pm 20}^{2}\left(\phi^{\prime}, \theta^{\prime}\right)\right\rangle=P \exp ( \pm 2 i \gamma) / \sqrt{6}
\end{gathered}
$$

are proportional to the scalar order parameter $S$ and the biaxiality parameter $P$.

By using the orthogonality conditions (A5) and Eqs. (A12)-(A14) we recover the relations in the traditional form [32]:

$$
\langle\mathbf{Q}(\hat{\mathbf{u}})\rangle_{\hat{\mathbf{u}}} \equiv \mathbf{S}(\hat{\mathbf{n}})=S \mathbf{Q}(\hat{\mathbf{n}})+P(\hat{\mathbf{m}} \otimes \hat{\mathbf{m}}-\hat{\boldsymbol{l}} \otimes \hat{\boldsymbol{l}}) / 2,
$$

$$
S=\left\langle 3(\hat{\mathbf{u}} \cdot \hat{\mathbf{n}})^{2}-1\right\rangle / 2,
$$

$$
P=3\left\langle(\hat{\mathbf{u}} \cdot \hat{\mathbf{m}})^{2}-(\hat{\mathbf{u}} \cdot \hat{l})^{2}\right\rangle / 2,
$$

where $\quad \hat{\mathbf{m}}=\cos \gamma \mathbf{e}_{x}(\hat{\mathbf{n}})-\sin \gamma \mathbf{e}_{y}(\hat{\mathbf{n}}) \quad$ and $\quad \hat{\boldsymbol{l}}=\sin \gamma \mathbf{e}_{x}(\hat{\mathbf{n}})$ $+\cos \gamma \mathbf{e}_{y}(\hat{\mathbf{n}})$.

The order parameter (A15) is a traceless symmetric tensor. Therefore, there are two nonvanishing independent invariants,

$$
\begin{gathered}
I_{2}=\operatorname{Tr}\left[\mathbf{S}^{2}(\hat{\mathbf{n}})\right]=\left(3 S^{2}+P^{2}\right) / 2, \\
I_{3}=\operatorname{Tr}\left[\mathbf{S}^{3}(\hat{\mathbf{n}})\right]=3 S\left(S^{2}-P^{2}\right) / 4,
\end{gathered}
$$

which enter the nonelastic part of the well known phenomenological expression for the Landau-de Gennes free energy density

$$
f_{\mathrm{LG}}=\frac{2 a}{3}\left(T-T^{*}\right) I_{2}-\frac{4 B}{3} I_{3}+\frac{4 C}{9} I_{2}^{2},
$$

where $T$ is the temperature and $T^{*}$ is the supercooling temperature.

For the scalar order parameters (A16) and (A17) combined into a pair $(S, P)$, it is convenient to introduce what might be called the "polar" (or amplitude-angle) representation

$$
S=s \cos \psi, \quad P=\sqrt{3} s \sin \psi,
$$

where $s^{2}=2 I_{2} / 3$. Using the representation (A21) the free energy density (A20) can be recast into the form

$$
f_{\mathrm{LG}}(s, \psi)=a\left(T-T^{*}\right) s^{2}-B s^{3} \cos (3 \psi)+C s^{4} \equiv B^{2} C^{-1} U_{\mathrm{LG}},
$$




$$
U_{\mathrm{LG}}(\eta, \psi)=\frac{8+t}{32} \eta^{2}-\eta^{3} \cos (3 \psi)+\eta^{4}, \quad \eta \equiv \frac{C}{B} s,
$$

where $t=32 a C B^{-2}\left(T-T_{c}\right)$ is the dimensionless temperature parameter and $T_{c}=T^{*}+B^{2} /(4 a C)$ is the temperature of the bulk nematic-isotropic transition. The rescaled density (A23) is a generalized version of the dimensionless free energy density previously used in Refs. [49,50].

Finally, we write down the components of the order parameter tensor (A16) in the polar representation,

$S_{i j}=s\left[n_{i} n_{j} \cos \psi+m_{i} m_{j} \cos (\psi-2 \pi / 3)+l_{i} l_{j} \cos (\psi+2 \pi / 3)\right]$,

and notice that the stationary points of the free energy density (A22) where the angle $\psi$ is a multiple of $\pi / 3$ represent uniaxially anisotropic states. The latter immediately recovers the well known result about the uniaxial anisotropy of NLC equilibrium states [57].

\section{APPENDIX B: TENSORIAL FORM OF INTERMOLECULAR POTENTIAL}

We begin with the intermolecular potential between two rigid, axially symmetric molecules expanded in a series of spherical harmonics as follows [58]:

$$
\begin{aligned}
U\left(\mathbf{r}, \hat{\mathbf{u}}_{1}, \hat{\mathbf{u}}_{2}\right)= & \sum_{j_{1}, j_{2}, j} u_{j_{1} j_{2} j}(r) \sum_{m_{1}, m_{2}, m} C_{m_{1} m_{2} m}^{j_{1} j_{2} j} \\
& \times Y_{j_{1} m_{1}}\left(\hat{\mathbf{u}}_{1}\right) Y_{j_{2} m_{2}}\left(\hat{\mathbf{u}}_{2}\right) Y_{j m}^{*}(\hat{\mathbf{r}})
\end{aligned}
$$

where $\mathbf{r} \equiv \mathbf{r}_{12}=\mathbf{r}_{1}-\mathbf{r}_{2} ; \mathbf{r}_{i}$ and $\hat{\mathbf{u}}_{i}$ are the position and orientation (equivalently, Euler angles of the long molecular axis) coordinates of the interacting molecules, respectively; $Y_{j m}(\hat{\mathbf{u}})=\sqrt{(2 j+1) /(4 \pi)} D_{m 0}^{j *}(\hat{\mathbf{u}})$ is the spherical function [55,59]. The form of the expansion (B1) implies that the potential is invariant under translations $\mathbf{r}_{i} \rightarrow \mathbf{r}_{i}+\Delta \mathbf{r}$, and rotations $\left\{\mathbf{r}_{i}, \hat{\mathbf{u}}_{i}\right\} \rightarrow\left\{R \mathbf{r}_{i}, R \hat{\mathbf{u}}_{i}\right\}$. In addition, we shall assume the head-tail symmetry

$$
U\left(\mathbf{r}, \hat{\mathbf{u}}_{1}, \hat{\mathbf{u}}_{2}\right)=U\left(\mathbf{r},-\hat{\mathbf{u}}_{1}, \hat{\mathbf{u}}_{2}\right)=U\left(\mathbf{r}, \hat{\mathbf{u}}_{1},-\hat{\mathbf{u}}_{2}\right),
$$

so that the outer sum in Eq. (B1) is restricted to run over even values of $j_{1}$ and $j_{2}$.

It is now our task to link the pairwise potential integrated over in-plane coordinates,

$$
\begin{aligned}
V\left(z, \hat{\mathbf{u}}_{1}, \hat{\mathbf{u}}_{2}\right)= & \int_{S} U\left(\mathbf{r}, \hat{\mathbf{u}}_{1}, \hat{\mathbf{u}}_{2}\right) d x d y \\
= & \sum_{j_{1}, j_{2}, j}\left[\left(2 j_{1}+1\right)\left(2 j_{2}+1\right)(2 j+1) /(4 \pi)^{3}\right]^{1 / 2} \\
& \times v_{j_{1} j_{2} j}(z) \sum_{m} C_{m-m 0}^{j_{1} j_{2} j} D_{m 0}^{j_{1}}\left(\hat{\mathbf{u}}_{1}\right) D_{-m 0}^{j_{2}}\left(\hat{\mathbf{u}}_{2}\right) D_{00}^{j}(\hat{\mathbf{z}}),
\end{aligned}
$$

and the tensorial representation that was originally suggested by Ronis and Rosenblatt in Ref. [36],

$$
\begin{aligned}
V\left(z, \hat{\mathbf{u}}_{1}, \hat{\mathbf{u}}_{2}\right) \equiv & V\left(z, \mathbf{Q}_{1}, \mathbf{Q}_{2}\right)=V_{\text {iso }}(z)+\beta^{(0)}(z) \hat{\mathbf{z}} \cdot\left(\mathbf{Q}_{1}+\mathbf{Q}_{2}\right) \cdot \hat{\mathbf{z}} \\
& +\beta^{(1)}(z) \operatorname{Tr}\left(\mathbf{Q}_{1} \mathbf{Q}_{2}\right)+\beta^{(2)}(z) \hat{\mathbf{z}} \cdot \mathbf{Q}_{1} \mathbf{Q}_{2} \cdot \hat{\mathbf{z}} \\
& +\beta^{(3)}(z)\left[\hat{\mathbf{z}} \cdot \mathbf{Q}_{1} \cdot \hat{\mathbf{z}}\right]\left[\hat{\mathbf{z}} \cdot \mathbf{Q}_{2} \cdot \hat{\mathbf{z}}\right],
\end{aligned}
$$

where $\mathbf{Q}_{i} \equiv \mathbf{Q}\left(\hat{\mathbf{u}}_{i}\right)$ is defined by Eq. (A11). In other words, the problem is to express the coefficients $\beta^{(i)}(z)$ in terms of the harmonics $v_{j_{1} j_{2} j}(z)$. To this end we restrict ourselves to the lowest order harmonics of the expansion (B3) with $j_{i}$ $<4$ and consider the equation

$$
\begin{array}{r}
x_{1}\left[\hat{\mathbf{z}} \cdot \mathbf{T}_{0}\left(\hat{\mathbf{u}}_{1}\right) \cdot \hat{\mathbf{z}}\right]\left[\hat{\mathbf{z}} \cdot \mathbf{T}_{0}\left(\hat{\mathbf{u}}_{2}\right) \cdot \hat{\mathbf{z}}\right]+x_{2} \operatorname{Tr}\left[\mathbf{T}_{0}\left(\hat{\mathbf{u}}_{1}\right) \mathbf{T}_{0}\left(\hat{\mathbf{u}}_{2}\right)\right] \\
\quad+x_{3} \hat{\mathbf{z}} \cdot \mathbf{T}_{0}\left(\hat{\mathbf{u}}_{1}\right) \mathbf{T}_{0}\left(\hat{\mathbf{u}}_{2}\right) \cdot \hat{\mathbf{z}}=\sum_{m=0}^{2} \alpha_{m} D_{m 0}^{2}\left(\hat{\mathbf{u}}_{1}\right) D_{-m 0}^{2}\left(\hat{\mathbf{u}}_{2}\right)
\end{array}
$$

that needs to be solved for $x_{1}, x_{2}$, and $x_{3}$. The sum on the right hand side of Eq. (B5) represents the sum of the harmonics with $j_{1}=j_{2}=2$. The case of the harmonics with $j_{1} j_{2}$ $=0$ is much easier to treat as we only have to use the relation

$$
\hat{\mathbf{z}} \cdot \mathbf{T}_{0}(\hat{\mathbf{u}}) \cdot \hat{\mathbf{z}}=D_{00}^{2}(\hat{\mathbf{u}}) .
$$

By using Eq. (A10) combined with the relations (A5) and (A6) it is straightforward to transform Eq. (B5) into a system of linear equations. The solution of the system is given by

$$
\begin{gathered}
x_{1}=\left(3 \alpha_{0}+4 \alpha_{1}+\alpha_{2}\right) / 2, \\
x_{2}=-2\left(\alpha_{1}+\alpha_{2}\right), \\
x_{3}=\alpha_{2} .
\end{gathered}
$$

Given the values of the Wigner coefficients, we can now use the relations (B6) and (B7) to derive the final result in the following form:

$$
\begin{gathered}
V_{\text {iso }}(z)=v_{000}(z) /(4 \pi)^{3 / 2}, \\
{\left[(4 \pi)^{3 / 2} / 5\right] \beta^{(0)}(z)=v_{202}(z)=v_{022}(z),} \\
{\left[(4 \pi)^{3 / 2} / 5\right] \beta^{(1)}(z)=2 /(3 \sqrt{5})\left[v_{220}(z)+10 / \sqrt{14} v_{222}(z)\right.} \\
\left.+3 / \sqrt{14} v_{224}(z)\right], \\
{\left[(4 \pi)^{3 / 2} / 5\right] \beta^{(2)}(z)=-20 / \sqrt{70}\left[v_{222}(z)+v_{224}(z)\right],} \\
{\left[(4 \pi)^{3 / 2} / 5\right] \beta^{(3)}(z)=35 / \sqrt{70} v_{224}(z) .}
\end{gathered}
$$

The formulas (B8)-(B12) relate the parameters of the representation (B4) to the coefficient functions in the spherical harmonics expansion (B3). The terms $v_{202}$ and $v_{022}$ describe the coupling between orientation of the molecules and the intermolecular vector, whereas $v_{220}$ and $v_{224}$ are known as the Maier-Saupe and the quadrupole terms, respectively. For the interaction between NLC molecules, the functions $\beta^{(1)}(z)$ and $\beta^{(2)}(z)$ define the elastic coefficients of NLC which must be positive. This stability condition implies that $\beta^{(1)}$ and $\beta^{(2)}$ are both predominantly nonpositive $[29,30,33,60]$. 
[1] T. J. Sluckin and A. Poniewierski, in Fluid Interfacial Phenomena, edited by C. A. Croxton (Wiley, Chichester, 1986), Chap. 5, pp. 215-253.

[2] B. Jérôme, Rep. Prog. Phys. 54, 391 (1991).

[3] G. Barbero and G. Durand, in Liquid Crystals in Complex Geometries, edited by G. P. Crawford and S. Žumer (Taylor \& Francis, London, 1996), Chap. 2, pp. 21-52.

[4] V. G. Chigrinov, Liquid Crystal Devices: Physics and Applications (Artech House, Boston, 1999).

[5] W. M. Gibbons, P. J. Shannon, S.-T. Sum, and B. J. Swetlin, Nature (London) 351, 49 (1991).

[6] M. Schadt, K. Schmitt, V. Kozinkov, and V. Chigrinov, Jpn. J. Appl. Phys., Part 1 31, 2155 (1992).

[7] A. Dyadyusha, T. Marusii, Y. Reznikov, V. Reshetnyak, and A. Khizhnyak, JETP Lett. 56, 17 (1992).

[8] M. O’Neill and S. M. Kelly, J. Phys. D 33, R67 (2000).

[9] S. Furumi, M. Nakagawa, S. Morino, and K. Ichimura, Appl. Phys. Lett. 74, 2438 (1999).

[10] H. G. Galabova, D. W. Allender, and J. Chen, Phys. Rev. E 55, 1627 (1996).

[11] S. Perny, P. L. Barny, J. Delaire, T. Buffeteau, C. Sourisseau, I. Dozov, S. Forget, and P. Martinot-Lagarde, Liq. Cryst. 27, 329 (2000).

[12] O. Yaroshchuk, T. Sergan, J. Kelly, and I. Gerus, Jpn. J. Appl. Phys., Part 1 41, 275 (2002).

[13] A. Petry, S. Kummer, H. Anneser, F. Feiner, and C. Bräuchle, Ber. Bunsenges. Phys. Chem. 97, 1281 (1993).

[14] N. C. R. Holme, P. S. Ramanujam, and S. Hvilsted, Appl. Opt. 35, 4622 (1996).

[15] L. Blinov, M. Kozlovsky, M. Ozaki, K. Skarp, and K. Yoshino, J. Appl. Phys. 84, 3860 (1998).

[16] Y. Wu, J.-I. Mamiya, O. Tsutsumi, A. Kanazawa, T. Shiono, and T. Ikeda, Liq. Cryst. 27, 749 (2000).

[17] O. Yaroshchuk, A. D. Kiselev, Y. Zakrevskyy, J. Stumpe, and J. Lindau, Eur. Phys. J. E 6, 57 (2001).

[18] O. Yaroshchuk, T. Sergan, J. Lindau, S. N. Lee, J. Kelly, and L.-C. Chien, J. Chem. Phys. 114, 5330 (2001).

[19] O. V. Yaroshchuk, A. D. Kiselev, Y. Zakrevskyy, T. Bidna, J. Kelly, L.-C. Chien, and J. Lindau, Phys. Rev. E 68, 011803 (2003).

[20] V. G. Chigrinov, V. M. Kozenkov, and H. S. Kwok, in Optical Applications in Photoaligning, edited by L. Vicari (Institute of Physics, Bristol, U.K., 2003), pp. 201-244.

[21] V. Chigrinov, E. Prudnikova, V. Kozenkov, H. Kwok, H. Akiyama, T. Kawara, H. Takada, and H. Takatsu, Liq. Cryst. 29, 1321 (2002).

[22] V. Chigrinov, A. Muravski, and H. S. Kwok, Phys. Rev. E 68, 061702 (2003).

[23] V. Chigrinov, S. Pikin, A. Verevochnikov, V. Kozenkov, M. Khazimullin, J. Ho, D. D. Huang, and H. S. Kwok, Phys. Rev. E 69, 061713 (2004).

[24] A. D. Kiselev, J. Phys.: Condens. Matter 14, 13417 (2002).

[25] L. T. Thieghi, R. Barberi, J. J. Bonvent, E. A. Oliveira, J. A. Giacometti, and D. T. Balogh, Phys. Rev. E 67, 041701 (2003).

[26] S. Oka, T. Mitsumoto, M. Kimura, and T. Akahane, Phys. Rev. E 69, 061711 (2004).

[27] O. V. Yaroshchuk, A. D. Kiselev, J. Lindau, V. Y. Reshetnyak,
A. G. Tereshchenko, and Y. A. Zakrevskyy, Ukr. Fiz. Zh. 46, 449 (2001).

[28] A. L. Alexe-Ionescu, R. Barberi, M. Iovane, and A. T. Ionescu, Phys. Rev. E 65, 011703 (2001).

[29] A. K. Sen and D. E. Sullivan, Phys. Rev. A 35, 1391 (1987).

[30] P. I. C. Teixeira and T. J. Sluckin, J. Chem. Phys. 97, 1490 (1992).

[31] P. I. C. Teixeira and T. J. Sluckin, J. Chem. Phys. 97, 1510 (1992).

[32] P. G. de Gennes and J. Prost, The Physics of Liquid Crystals (Clarendon Press, Oxford, 1993).

[33] M. A. Osipov and S. Hess, J. Chem. Phys. 99, 4181 (1993).

[34] M. A. Osipov and T. J. Sluckin, J. Phys. II 3, 793 (1993).

[35] M. A. Osipov, T. J. Sluckin, and S. J. Cox, Phys. Rev. E 55, 464 (1997).

[36] D. Ronis and C. Rosenblatt, Phys. Rev. A 21, 1687 (1980).

[37] W. Zhao, C.-X. Wu, and M. Iwamoto, Phys. Rev. E 62, R1481 (2000).

[38] W. Zhao, C.-X. Wu, and M. Iwamoto, Phys. Rev. E 65, 031709 (2002).

[39] Y. Guo-Chen, Z. Shu-Jing, H. Li-Jun, and G. Rong-Hua, Liq. Cryst. 31, 1093 (2004).

[40] I. F. Lyuksyutov, Sov. Phys. JETP 48, 178 (1978).

[41] E. Penzenstadler and H.-R. Trebin, J. Phys. (France) 50, 1027 (1989).

[42] R. Rosso and E. Virga, J. Phys. A 29, 4247 (1996).

[43] S. Kralj and E. Virga, J. Phys. A 34, 829 (2001).

[44] Y. Iimura, N. Kobayashi, and S. Kobayashi, Jpn. J. Appl. Phys., Part 1 34, 1935 (1995).

[45] V. P. Vorflusev, H.-S. Kitzerow, and V. Chigrinov, Jpn. J. Appl. Phys., Part 2 34, L1137 (1995).

[46] H. Yokoyama and H. A. van Sprang, J. Appl. Phys. 57, 4520 (1985).

[47] Y. A. Nastishin, R. D. Polak, S. V. Shiyanovskii, V. H. Bodnar, and O. D. Lavrentovich, J. Appl. Phys. 86, 4199 (1999).

[48] H. Yokoyama and R. Sun, Jpn. J. Appl. Phys., Part 2 39, L45 (2000).

[49] P. Sheng, B.-Z. Li, M. Zhou, T. Moses, and Y. R. Shen, Phys. Rev. A 46, 946 (1992).

[50] T. Z. Qian and P. Sheng, Phys. Rev. E 55, 7111 (1997).

[51] A. Murauski, V. Chigrinov, A. Muravsky, Fion Sze-Yan Yeung, J. Ho, and H.-S. Kwok, Phys. Rev. E 71, 061707 (2005).

[52] T. J. Sluckin and P. Shukla, J. Phys. A 16, 1539 (1983).

[53] J. W. Cahn, J. Chem. Phys. 66, 3667 (1977).

[54] P. G. de Gennes, Rev. Mod. Phys. 57, 827 (1985).

[55] L. C. Biedenharn and J. D. Louck, Angular Momentum in Quantum Physics (Addison-Wesley, Reading, MA, 1981).

[56] I. M. Gelfand, R. A. Minlos, and Z. Y. Shapiro, Representations of Rotation and Lorenz Groups and Their Applications (Pergamon Press, Oxford, 1963).

[57] A. Z. Patashinskii and V. L. Pokrovskii, Fluctuational Theory of Phase Transitions, 2nd ed. (Nauka, Moscow, 1982) (in Russian).

[58] J. A. Pople, Proc. R. Soc. London, Ser. A 221, 498 (1954).

[59] Handbook of Mathematical Functions, edited by M. Abramowitz and I. A. Stegun (Dover, New York, 1972).

[60] B. Tjipto-Margo and D. E. Sullivan, J. Chem. Phys. 88, 6620 (1988). 